\title{
L-ergothioneine and Metformin Alleviates Liver Injury in Experimental Type-2 Diabetic Rats via Reduction of Oxidative Stress, Inflammation, and Hypertriglyceridemia
}

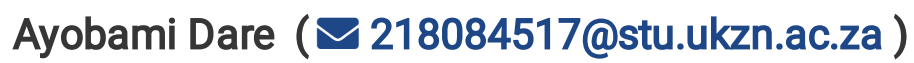

University of KwaZulu-Natal College of Health Sciences

Mahendra Channa

University of KwaZulu-Natal College of Health Sciences

Anand Nadar

University of KwaZulu-Natal College of Health Sciences

\section{Research Article}

Keywords: Antioxidant, diabetes, L-ergothioneine, cytoprotection, Liver

Posted Date: May 24th, 2021

DOI: https://doi.org/10.21203/rs.3.rs-480961/v1

License: (c) (i) This work is licensed under a Creative Commons Attribution 4.0 International License.

Read Full License 


\section{Abstract}

Diabetic-induced liver toxicity is a serious complication that cause significant metabolic dysfunction. Lergothioneine (L-egt) is a bioactive nutraceutical obtained from mushrooms and certain food products, with reported cytoprotective, antioxidant and anti-inflammatory properties and potential to improve efficacy of existing therapy. Thus, this study evaluates the effects of L-egt, and/or metformin, on diabetes-induced liver injury. Diabetes was induced in male Sprague-Dawley rats using $10 \%$ fructose for two weeks, followed by a single low dose streptozotocin (STZ, $40 \mathrm{mg} / \mathrm{kg}$ i.p) injection. After induction of diabetes, animals were treated either with de-ionized water (DW), L-egt (35 mg/kg bwt), metformin (500 $\mathrm{mg} / \mathrm{kg}$ bwt), or a combination of L-egt and metformin orally for seven weeks. Body weight and glucose were monitored during the experiment. At the completion of experiment, blood samples were collected, and liver tissue was excised for biochemical analysis, enzyme-link immunosorbent assay (ELISA) of various liver function biomarkers, reverse transcription-quantitative polymerase chain reaction (RT-qPCR) analysis of genes associated with inflammation, oxidative stress, and lipid metabolism, as well as histopathological evaluation. Diabetic animals showed liver hypertrophy, increased liver injury, hepatic triglycerides, oxidative stress, and hepatic inflammation. However, L-egt, and/or metformin, improved glycemic control, reduced liver injury, triglycerides, oxidative damage, inflammatory injury, and normalize liver histology by upregulating Nrf2/Sirt1, downregulate NF-kB/TGF-B1, and reduce SREBP1c/FAS expression. In conclusion, these results showed that combination of L-egt and metformin improve therapeutic efficacy than either treatment alone. Thus, L-egt can be used as an adjuvant to mitigate diabetes-induced liver complication.

\section{Introduction}

Type-2 diabetes (T2D), a chronic metabolic disorder of global prevalence, is drastically increasing in developing and industrialized countries due to excessive caloric intake, sedentary lifestyle, poor diagnosis, and disease management. The etiology and progression of T2D are multifaceted and frequently associated with obesity, hypertriglyceridemia, insulin resistance (IR), and compromised insulin secretion by the pancreatic islet with subsequent hyperglycemia (Lozano et al., 2016, Pandey et al., 2015). Liver complications are often seen in approximately $70 \%$ of people with diabetes and account for about 2-4\% mortality in T2D patients (Hazlehurst et al., 2016, Zoppini et al., 2014). These complications (including non-alcoholic fatty liver disease, steatosis) results from hyperglycemia-induced oxidative injury and low-grade inflammation that mediate liver damage, including fibrosis and apoptosis. Also, IR increases de novo lipogenesis under the influence of SREBP-1 $c$ and alters lipid metabolism by upregulating FAS, thereby promoting the influx of fatty acid into the liver cells (Perry et al., 2015, Gehrke and Schattenberg, 2020, Arrese et al., 2019). Excessive accumulation and infiltration of fats into the hepatocytes contribute to liver toxicity by increasing production of reactive oxygen species (ROS), stimulate inflammatory cytokines, mitochondrial dysfunction, and endoplasmic reticular stress that mediate liver damage. Thus, therapeutic measures that can effectively inhibit oxidative injury, prevent inflammation, and reduce fatty infiltration into the hepatocytes may provide adequate cytoprotection 
against liver damage. However, recent attention has been geared towards natural compounds with multiple bioactivities to provide adjuvant therapy to manage diabetic liver complications.

The beneficial role of bioactive compounds with antioxidants properties in the management of diabetic complications has been associated with their ability to increase antioxidant capacity and stimulate endogenous antioxidant enzymes and cytoprotective genes, activating various transcription factors such as Nrf2 and Sirt1 (Jadeja et al., 2016, Farghali et al., 2019). These compounds also exert antiinflammatory activities by downregulating the Nuclear factor-kappa B signaling pathway to inhibit the production of cytokines, chemokines, and fibrosis in the liver (de Gregorio et al., 2020, McKinley and Willoughby, 2014). Furthermore, natural compounds also help reduce serum cholesterol and triglycerides by downregulating the expression of lipogenic proteins, thereby reducing the influx of triglyceride into hepatocytes (Xu et al., 2020, Romano et al., 2021). Thus, attention has been geared towards nutraceuticals that regulate glycemic control, upregulate the antioxidant pathway, inhibit cellular inflammation and improve lipid metabolism in the management of diabetic complications (Wang et al., 2018, Ma et al., 2019). Recently, the therapeutic benefits of mushrooms in the management of diabetes and associated complications have received significant attention owing to the presence of various bioactive compounds, including flavonoids and L-ergothioneine (Lo et al., 2020, Lindequist and Haertel, 2020, Azeem et al., 2021).

L-ergothioneine (L-egt), an adaptive antioxidant obtained from mushroom and some meat products (e.g., kidney and liver), has been shown to exert antioxidant and anti-inflammatory activities as well as exhibit adaptive cytoprotective function by accumulating at the site of injury to protect against tissue damage (Halliwell et al., 2018, Ey et al., 2007, Salama and Omar, 2021, Ko et al., 2021). L-egt protects against hyperglycemia-induced cell senescence, prevents embryo malformations in diabetic pregnant rats, and enhances the therapeutic efficacies in-vitro and in-vivo (Song et al., 2017, D'Onofrio et al., 2016, Guijarro et al., 2002). Furthermore, administration of L-egt rich foods ameliorated dimethylnitrosamine-induced liver fibrosis and oxidative stress in mice (Tang et al., 2016) and has been shown in the treatment of nonalcoholic fatty liver disease (NAFLD) to downregulate SREBP1c and FAS expression, thereby inhibiting hepatic lipogenesis and lipid accumulation (Carbonero et al., 2019, Jeong and Park, 2020). Also, the hepatoprotective effect of biguanides, e.g., Metformin, may result from its potency to regulate glycemic index by reducing hepatic gluconeogenesis and increased glucose uptake (Rena et al., 2017). However, metformin is associated with side effects, and patients still present with liver complications despite glycemic control, suggesting that metformin alone does not confer overall effective treatment. Therefore, this study evaluated the role of L-egt, with or without metformin, on liver injury in a rat model of type-2 diabetes.

\section{Material And Methods}

\section{Drugs and chemicals}


Pure L-egt was obtained from Tetrahedron limited, Paris, France. QPCR iTAQ SYBR Green and cDNA synthesis kits were purchased from Lasec (Cape Town, South Africa). Primers were synthesized by Inqaba Biotec (Pretoria, South Africa). Metformin was obtained from a local pharmacy (Pharmed, South Africa). All other chemicals, reagents, and equipment were procured from standard commercial suppliers and high analytical grades.

\section{Experimental animals and ethical approval}

Thirty-six (36) male Sprague-Dawley rats $(175 \pm 20 \mathrm{~g})$ were obtained from the Biomedical Research Unit, Westville Campus, University of KwaZulu-Natal (UKZN), South-Africa and were housed in a room with standard laboratory conditions (12 hours light-dark cycles; temperature $23 \pm 1^{\circ} \mathrm{C}, 40-60 \%$ humidity). The animals were allowed access to rat feed and water ad libitum for an acclimatization period of one week before the experiment. All animal and experimental procedures were approved by the Animal Research Ethics Committee (AREC) of the University of KwaZulu-Natal, Durban, South Africa (Ethic number: AREC/006/019D).

\section{Experimental design}

After acclimatization, the animals were randomly divided into two major groups: the non-diabetic $(n=10)$ and the diabetic $(n=20)$ groups. All animals in the diabetic group were treated with fructose and streptozotocin (STZ) to induce type-2 diabetes using the established model described by (Wilson and Islam, 2012). Briefly, the animals were supplied $10 \%$ fructose in drinking water ad-libitum for two weeks to induce IR and later injected (i.p.) $40 \mathrm{mg} / \mathrm{kg}$ bwt STZ freshly prepared in $0.1 \mathrm{M}$ citrate buffer. The animals in the non-diabetic group were injected with the same volume of $0.1 \mathrm{M}$ citrate buffer. Animals with nonfasting blood glucose levels of $>16.7 \mathrm{mmol} / \mathrm{L}$ after one-week post-STZ injection were confirmed diabetic (Srinivasan et al., 2005) and included in the study. After successful diabetes induction, the non-diabetic animals were subdivided into two groups, while the diabetic animals were subdivided into four groups as follows.

Group 1 (NC): non-diabetic plus de-ionized water (negative control).

Group-2 (NE): non-diabetic plus L-egt

Group-3 (DC): diabetic plus de-ionized water (positive control)

Group-4 (DE): diabetic plus L-egt

Goup-5 (DM): diabetic plus metformin

Group-6 (DEM): diabetic plus l-egt plus Metformin.

Groups 1 and 3 were administered de-ionized water $(1 \mathrm{ml} / 100 \mathrm{~g})$, groups 2 and 4 were administered L-egt (35mg/kg bwt), group 5 was administered metformin $(500 \mathrm{mg} / \mathrm{kg}$ bwt), while group 6 was administered a combination of L-egt and metformin. The dosage of L-egt used in this study was based on previous in- 
vivo studies using this nutraceutical (Tang et al., 2018, Williamson et al., 2020). All treatments were done daily by oral gavage and lasted for seven weeks.

\section{Blood and tissue collection}

After the seven-week treatment period, all animals were sacrificed by decapitation and blood was immediately collected into a serum vacutainer EDTA bottle and allowed to stand for 30 mins. The blood was then centrifuged at $3000 \mathrm{rpm}$ for $10 \mathrm{mins}$ at $4^{0} \mathrm{C}$ to obtain serum. The serum samples obtained were stored in the bio-freezer (Snijers Scientific, Holland) at $-80^{\circ} \mathrm{C}$ until used for biochemical analysis.

Afterward, incisions were made along the linea alba of the anterior abdominal wall to excise the liver. This organ weighed, rinsed with cold normal saline, and snap-frozen in liquid nitrogen before been stored in the bio-freezer at $-80^{\circ} \mathrm{C}$ until used for analysis. Liver tissue was fixed in $10 \%$ neutral-buffered formalin for histological assessment.

\section{Analysis of Bodyweight and liver index}

Body weight was monitored using a sensitive electronic weighing scale (Metler, Greifensee, Switzerland). The liver index (use to assess liver hypertrophy) was calculated as the ratio of harvested liver weight to the body weight and expressed in percentage i.e.

Liver index $=$ Liver weight $\times 100$

Body weight

\section{Preparation of liver homogenates}

The liver was thawed and homogenized in $10 \%$ phosphate buffer $(0.1 \mathrm{M}, \mathrm{pH} 7.4,1: 9 \mathrm{w} / \mathrm{v})$. The homogenates were centrifuged a $600 \mathrm{~g}$ for $10 \mathrm{~min}$ to remove cell debris. The supernatant was subsequently centrifuged at $10,000 \mathrm{~g}$ for $20 \mathrm{mins}$ to obtain the cytosolic fraction, which was used immediately for biochemical analyses.

\section{Biochemical analysis}

The serum concentration of liver enzymes (AST, ALT, and ALP) and triglyceride were analyzed at an accredited pathology laboratory (Global Clinic and Viral laboratories, Amazimtoti, South Africa). The concentration of triglyceride was also measured in the liver homogenate using an automatic biochemical analyzer. Blood glucose was measured at the end of the experiment using a glucometer (Accu-Chek Performa, USA). Serum insulin levels were measured by ELISA kits (Mercodia kit), and used to evaluate homeostasis model assessment of insulin resistance (HOMA-IR), which was calculated as previously described by (Matthews et al., 1985) using the following formula:

HOMA-IR = fasting serum insulin $(\mathrm{mU} / \mathrm{L}) \mathrm{X}$ fasting blood glucose $(\mathrm{mg} / \mathrm{dl})$. 


\section{Analysis of lipid peroxidation (MDA), antioxidant enzymes (SOD and CAT), and reduced glutathione level (GSH)}

The liver homogenates were used to measure the concentration of malondialdehyde (MDA), superoxide dismutase (SOD), catalase (CAT), and reduced glutathione (GSH) by spectrophotometric assay. MDA, a marker of lipid peroxidation, was evaluated by measuring the content of thiobarbituric acid (TBA) reactive product in the liver homogenates using the method of Mkhwanazi et al. (Mkhwanazi et al., 2014) and expressed as nmol MDA per milligram protein. GSH level and SOD activity were assessed using the method of Ellman and Marklund, respectively (Ellman, 1959, Marklund, 1985), while CAT activity was assessed using the protocol of Aebi (Aebi, 1984).

\section{Analysis of inflammatory biomarkers}

The concentration of cytokine- tumor necrosis factor- $a(T N F-a)$; chemokine, monocyte chemotactic protein-1 (MCP-1) and fibrotic cytokine, tumor growth factor- $\beta$ (TGF- $\beta$ ) were quantified in the liver homogenates using Elabscience commercial ELISA kits according to the manufacturer's protocol.

\section{Rt-PCR Analysis of SREBP1c, FAS, Nrf2, Sirt1, NF-kB and TGF- $\beta 1$ mRNA expression}

The relative mRNA expression of SREBP1c, FAS, Nrf2, Sirt1, NF-kB, and TGF- $\beta 1$ was quantified in the liver homogenates and analyzed using a light cycler. Total RNA was isolated in the liver using TRIzol reagent (40mg of tissue/mL, Trizol reagent). The isolated RNA quantity was determined by measuring absorbance at 260/280nm using nanodrop ND-1000 spectrophotometer (Thermo scientific, Johannesburg, South Africa). The total RNA was converted into cDNA using iScript cDNA synthesis kit, Life Science research (Biorad, South Africa) following manufacturers' instruction. The complete reaction mixture was incubated on SimpliAmp ${ }^{T M}$ thermal cycler, Applied Biosystems (Thermo Fischer Scientific), using the following reaction condition; priming 5 mins at $25^{\circ} \mathrm{C}$, reverse transcription $20 \mathrm{mins}$ at $46^{\circ} \mathrm{C}$, and RT inactivation $1 \mathrm{~min}$ at $95^{\circ} \mathrm{C}$. Real-time polymerase chain reaction (RT-PCR) was done using iTaq Universal SYBR Green supermix (Biorad, CA, USA) as fluorescent dye on a light cycler 96 RT-PCR system (Roche, Mannheim, Germany). RT-qPCR was performed in a $10 \mu \mathrm{L}$ reaction volume containing $5 \mu \mathrm{L}$ SYBR Green Master Mix, $1 \mu \mathrm{L}$ of each primer, $1 \mu \mathrm{L}$ of nuclease-free water, and $2 \mu \mathrm{L}$ of cDNA template. The primer sequences used are provided in Table-1. The purity and specificity of amplified PCR products were verified by melting curves generated at the end of each PCR. Relative mRNA expression was calculated using the $2^{-\Delta \Delta c t}$ method (Livak and Schmittgen, 2001) and normalized in relation to the endogenous control expression, GAPDH. The primers sets were homology searched using an NCBI BLAST search to ensure that they were specific.

Table-1: Oligonucleotide sequence and accession number 


\begin{tabular}{|c|c|c|}
\hline Genes & Primer sequence & Genbank Accession number \\
\hline \multirow[t]{2}{*}{ GAPDH } & F:5'-TGATGACATCAAGAAGGTGGTGGAG-3' & XM_017593963.1 \\
\hline & R: 5'-TCCTTGGAGGCCATGTAGGCCAT-3' & \\
\hline \multirow[t]{2}{*}{$\mathrm{Nrf}_{2}$} & F: 5'-CAGCATGATGGACTTGGAATTG - 3' & NM_031789.2 \\
\hline & R: 5'-GCAAGCGACTCATGGTCATC - 3' & \\
\hline \multirow[t]{2}{*}{ Srt1 } & F: 5'-CCCAGATCCTCAAGCCATGTTC - 3' & NM_001372090.1 \\
\hline & R: 5'-TGTGTGTGTGTTTTTCCCCC - 3' & \\
\hline \multirow[t]{2}{*}{ TGF- $\beta 1$} & F: 5'-GGGCTACCATGCCAACTTCTG - 3' & NM_021578.2 \\
\hline & R: 5'-GAGGGCAAGGACCTTGCTGTA-3' & \\
\hline \multirow[t]{2}{*}{ NF-kB } & F: 5'-ACGATCTGTTTCCCCTCATCT-3' & NM_199267.2 \\
\hline & R: 5'-TGCTTCTCTCCCCAGGAATA-3' & \\
\hline \multirow[t]{2}{*}{ FAS } & F: 5'-TGTGGGGTGGAAATCATCGG-3' & NM_012820.1 \\
\hline & R: 5'-CATTGCTCCTTTGGGGTTGC-3' & \\
\hline \multirow[t]{2}{*}{ SREBP-1C } & F: 5'- GGAGCCATGGATTGCACATT-3' & NM_001276708.1 \\
\hline & R: 5'- AGGAAGGCTTCCAGAGAGGA-3' & \\
\hline
\end{tabular}

\section{Histopathological analysis of Liver}

Liver specimens were embedded in paraffin wax after dehydration in a graded series of ethanol and cleared in xylene. Serial sections were done using a rotary microtome; liver slices of 5- $\mu \mathrm{m}$ thick were fixed on a slide and stained with hematoxylin \&eosin (H\&E). The stained sections were visualized and captured using a nanozoomer S360 digital slide scanner (Hamamatsu Photonics, Japan) and nanozoomer digital pathology version 2.8 software for analysis by a pathologist.

\section{Statistical analysis}

Data were reported as mean \pm SEM. GraphPad Prism Software version 7 (San Diego, CA) was used for statistical analysis. The differences between means were analyzed using a one-way analysis of variance (ANOVA) followed by Tukey's multiple comparison test to determine the difference between groups. Statistical significance between groups was considered at $P<0.05$.

\section{Results}

\section{Effect body weight, liver hypertrophy, blood glucose, HOMA- IR, and serum TG in T2DM-rats}


As presented in table 2 , the DC group had a significant reduction $(p<0.001)$ in body weight vs. NC. Coadministration of L-egt with metformin in diabetic rats caused a significant increase $(D E: p<0.05)$ in body weight vs. DC. There was a significant increase $(p<0.001)$ in the liver hypertrophy of DC rats vs. NC rats. However, L-egt, with or without metformin to diabetic rats significantly reduced (DE: $p<0.05 ; D E M: p$ $<0.01)$ liver hypertrophy vs. DC. There was a significant increase $(p<0.001)$ in blood glucose level in the DC group vs NC group. Co-administration of L-egt with metformin significantly reduced $(p<0.01)$ blood glucose level vs. DC. There was a significant increase in HOMA-IR $(p<0.01)$ in the DC group vs NC group. However, combination of L-egt with metformin significantly reduced HOMA-IR $(p<0.05)$ vs. DC. Serum TG increased significantly $(p<0.01)$ in the DC rats vs. NC rats, while L-egt alone, and its combination with metformin, significantly reduced (DE: $p<0.05$; DEM: $p<0.01$ ) serum TG vs. DC rats.

Table-2: Effect of L-egt with or without metformin for seven weeks on body weight, liver index, blood glucose and HOMA-IR in T2DM-rats. ${ }^{* *} p<0.01,{ }^{* \star *} p<0.001$ vs NC; ${ }^{*} p<0.05,{ }^{\# \#} p<0.01$, vs DC. $(n=5)$. NC= Nondiabetic control; NE = nondiabetic plus L-egt; $D C=$ diabetic control; $D E=$ diabetic plus L-egt; $D M=$ diabetic plus metformin and DEM = diabetic plus L-egt plus metformin. (supplemental data)

\begin{tabular}{|lllllll|}
\hline Indices & NC & NE & DC & DE & DM & DEM \\
\hline Body weight (g) & $\begin{array}{l}431.20 \pm \\
16.68\end{array}$ & $\begin{array}{l}424.20 \pm \\
15.85\end{array}$ & $277.80 \pm$ & $321.40 \pm$ & $338.60 \pm$ & $358.60 \pm$ \\
& & $20.68^{\star \star \star}$ & 8.44 & 15.79 & $12.97^{\#}$ \\
Liver index (\%) & $3.11 \pm$ & $3.17 \pm$ & $4.28 \pm$ & $3.49 \pm$ & $3.35 \pm$ & $3.28 \pm$ \\
& 0.08 & 0.08 & $0.24^{\star \star \star}$ & $0.23^{\#}$ & $0.08^{\# \#}$ & $0.17^{\# \#}$ \\
Blood glucose & $80.64 \pm$ & $73.08 \pm$ & $257.34 \pm$ & $214.44 \pm$ & $175.90 \pm$ & $143.54 \pm$ \\
(mg/dl) & 7.64 & 8.57 & $28.15^{\star \star \star}$ & 23.02 & $20.87^{\#}$ & $12.96^{\# \#}$ \\
HOMA-IR & $2.51 \pm$ & $2.39 \pm$ & $5.04 \pm$ & $3.94 \pm$ & $3.77 \pm 0.45$ & $3.07 \pm 0.37^{\#}$ \\
& 0.42 & 0.47 & $0.46^{\text {\# }}$ & 0.45 & & \\
\hline TG (mmol/L) & $1.47 \pm$ & $0.97 \pm$ & $2.88 \pm$ & $1.78 \pm$ & $1.84 \pm$ & $1.65 \pm$ \\
& 0.17 & 0.19 & $0.30^{\star \star}$ & $0.13^{\#}$ & $0.17^{\#}$ & $0.16^{\# \#}$ \\
\hline
\end{tabular}

\section{Effect on liver injury in T2DM rats.}

The effects of L-egt treatment with or without metformin for seven weeks on biomarkers of liver injury (ALP, AST, and ALT) and liver triglyceride in type-2 diabetic rats were presented in table-3. In the DC group, liver injury biomarkers increased significantly (ALP: $p<0.05$; AST: $p<0.001$ and ALT: $p<0.001$ ) vs. the NC group. However, L-egt, with or without metformin, significantly reduced (DE: $p<0.05$; DEM: $p<0.01$ ) the concentration of liver biomarkers vs. DC. Liver triglyceride significantly $(p<0.01)$ increase in the DC group vs. NC group while L-egt, with or without metformin cause a significant decrease $(p<0.05)$ in hepatic triglyceride level vs. DC. 
Table 3

Effect of L-egt with or without metformin on liver enzymes, and triglycerides in T2DM-rats. ${ }^{* \star *} \mathrm{p}<0.001$,

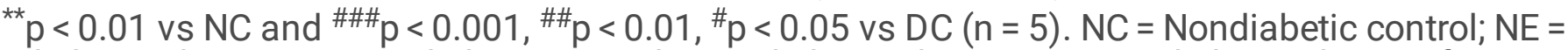
nondiabetic plus L-egt; $\mathrm{DC}=$ diabetic control; $\mathrm{DE}=$ diabetic plus L-egt; $\mathrm{DM}=$ diabetic plus metformin and DEM = diabetic plus L-egt plus metformin. (supplemental data).

\begin{tabular}{|lllllll|}
\hline Indices & NC & NE & DC & DE & DM & DEM \\
\hline ALP (U/L) & $129.60 \pm$ & $124.20 \pm$ & $239.60 \pm$ & $147.40 \pm$ & $141.60 \pm$ & $132.60 \pm$ \\
& 15.58 & 24.00 & $29.04^{\star \star}$ & $16.33^{\#}$ & $10.26^{\#}$ & $11.61^{\# \#}$ \\
AST (U/L) & $87.40 \pm$ & $89.20 \pm$ & $184.20 \pm$ & $127.20 \pm$ & $126.00 \pm$ & $97.40 \pm$ \\
& 11.21 & 14.18 & $14.51^{\star \star \star}$ & $9.88^{\#}$ & $9.82^{\#}$ & $15.22^{\# \# \#}$ \\
ALT (U/L) & $87.20 \pm$ & $86.80 \pm$ & $172.04 \pm$ & $122.40 \pm$ & $122.20 \pm$ & $105.80 \pm$ \\
& 9.28 & 14.97 & $10.35^{\star \star \star}$ & $11.41^{\#}$ & $7.31^{\#}$ & $11.82^{\# \#}$ \\
TG (mmol//g & $1.17 \pm$ & $0.89 \pm$ & $3.29 \pm$ & $1.84 \pm$ & $1.85 \pm$ & $1.80 \pm 0.28^{\#}$ \\
liver) & 0.23 & 0.19 & $0.35^{\star \star}$ & $0.36^{\#}$ & $0.33^{\#}$ & \\
\hline
\end{tabular}

\section{Effect on lipid peroxidation and antioxidant enzymes}

As presented in Fig. 1a-d, liver MDA concentration in the DC group was significantly increased $(p<0.001)$ vs. the NC group. Administration of L-egt alone and its co-treatment with metformin to diabetic rats caused a significant decrease (DC: $p<0.05$; DEM: $p<0.01$ ) in liver MDA concentration vs. the DC group. There was a significant decrease $(P<0.01)$ in SOD, GSH, and CAT in the DC rats vs. NC. However, L-egt, with or without metformin, significantly increase SOD, CAT (DE: $p<0.05$; DEM: $p<0.01)$ and GSH $(p<$ $0.05)$ in the diabetic rat vs. DC.

Fig-1 c: GSH levels in L-egt, with or without metformin, treated T2DM rats

Figure 1a-d

Effect of L-egt, with or without metformin on Lipid peroxidation, antioxidant enzymes and glutathione in T2DM-rats. ${ }^{* \star} p<0.001,{ }^{* *} p<0.01$ vs NC; ${ }^{\# \#} p<0.01,{ }^{\#} p<0.05$ vs DC. $(n=5) . N C=$ Nondiabetic control; $N E=$ nondiabetic plus L-egt; $D C=$ diabetic control; $D E$ = diabetic plus L-egt; $D M=$ diabetic plus metformin and $\mathrm{DEM}=$ diabetic plus L-egt plus metformin (supplemental data).

\section{Effects on liver inflammatory biomarkers: TNF-叉, MCP-1, and TGF- $\beta 1$.}

The concentration of TNF- $\alpha$, MCP-1, and TGF- $\beta 1$ in the liver homogenates after treatment with L-egt, with or without metformin, for seven weeks, was presented in Fig. 2a-c. DC group had a significant increase (MCP1: $p<0.01$; TNF-a: $p<0.001$; TGF- $\beta 1$ : $p<0.01$ ) in the concentration of inflammatory biomarkers vs. NC group. Diabetic groups treated with L-egt alone, or in combination with metformin caused a significant reduction in MCP1, TGF- $\beta 1(p<0.05)$, and TNF-a (DE: $p<0.05$; DEM: $p<0.01)$ vs. DC group. 
Figure 2a-b: Effect of L-egt, with or without metformin, on liver inflammatory biomarkers (A) MCP1, (B) TNF- $\alpha$ and TGF- $\beta 1$ in T2DM-rats. ${ }^{* \star *} p<0.001,{ }^{* *} p<0.01$ vs NC; ${ }^{\# \#} p<0.01,{ }^{\#} p<0.05$ vs DC. $(n=5)$. NC= Nondiabetic control; NE = nondiabetic plus L-egt; $\mathrm{DC}=$ diabetic control; $\mathrm{DE}=$ diabetic plus L-egt; $\mathrm{DM}=$ diabetic plus metformin and DEM = diabetic plus L-egt plus metformin. (supplemental data).

\section{Effect on liver mRNA relative expression of SREBP1c, FAS, NF-kB, TGFB1, Nrf2, and Sirt1}

The transcriptional levels of liver SREBP1c, FAS, NF-kB, $(p<0.001)$ and TGF- $\beta 1(p<0.01)$ significantly increase in the DC group vs. NC group. Administration of L-egt, with or without metformin, to diabetic rats significantly reduced the expression of SREBP1c (DE: $p<0.05$; DEM: $p<0.01)$, FAS (DEM: $p<0.01), N F-k B$ (DE: $p<0.05$; DEM: $p<001)$ and TGF- $\beta 1$ ( $p<0.05)$ vs. DC rats, (Fig. 3A and B). There was a significant decrease in mRNA expression of liver Nrf2 and Sirt1 $(p<0.01)$ in the DC rats vs. NC rats (Fig. 3C). However, administration of L-egt, with or without metformin to diabetic rats significantly increased (DE: $p$ < 0.05; DEM: $p$ < 0.01) Nrf2 and Sirt1 mRNA expression vs. DC rats. Interestingly, co-administration of Legt with metformin to diabetic rats (DEM) significantly $(p<0.05)$ increased Nrf2 mRNA expression vs. diabetic rats treated with metformin alone (DM).

Figure 3a-c: Relative mRNA expression level of (A) SREBP1c and FAS (B), NF-kB and TGF- $\beta 1$ (C), Nrf2 and Sirt1 relative gene expression in liver tissues of diabetic animals treated with L-egt with or without

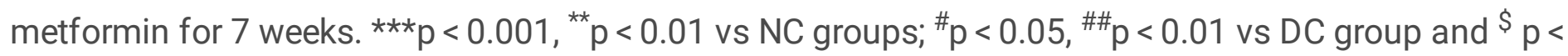
0.05 vs $\mathrm{DM}(\mathrm{n}=5)$. NC = Nondiabetic control; $\mathrm{NE}=$ nondiabetic plus L-egt; $\mathrm{DC}=$ diabetic control; $\mathrm{DE}=$ diabetic plus L-egt; DM = diabetic plus metformin and DEM = diabetic plus L-egt plus metformin (supplemental data).

\section{Effect on liver histopathological changes.}

Morphological evaluation of the liver sections after seven weeks of treatment showed that L-egt, with or without metformin, alleviates hepatic injury in T2DM animals, as shown in Fig. 4a-f. The photomicrograph of liver sections in NC and NE groups showed normal liver histoarchitecture with normal morphology of the central vein and hepatic sinusoids (Fig. 4a and b). The liver section in the DC animal (Fig. 4c) showed liver injury characterized by disrupted hepatic sinusoids, congested central vein with mild hepatocyte degeneration compared to the NC rats. Administration of L-egt (DE) or metformin (DM), reduced sinusoid disruption and congestion of the central vein (Fig. $4 \mathrm{~d}$ and $4 \mathrm{e}$ resp). In contrast, the liver section in DEM animals shows similar histoarchitecture with NC animals (Fig. 5f).

\section{Discussion}

This study aimed to examine the benefits of L-ergothioneine, with or without metformin, on liver injury in a type-2 diabetic rat model. Both hyperglycemia and IR increase the production of reactive oxygen species (ROS) that alter the structure and functions of vital organs (including the liver) with the resultant pathogenesis of diabetic complications. In this regard, the use of natural compounds with significant 
bioactive potential have attracted greater interest due to their reduced side effect, increased accessibility and efficacy against the molecular and cellular triggers involved in diabetic complications (Choudhury et al., 2017, Gothai et al., 2016).

A significant reduction in body weight has been reported in poorly managed diabetes (Magalhães et al., 2019). A similar observation was reported in this study where the DC rats show substantial weight reduction compared to NC. This reduction could be correlated with metabolic derangements associated with poor glucose utilization, thereby resulting in excess catabolism of adipose tissue, breakdown of structural proteins and reduced protein synthesis in all tissues, thereby causing muscle wasting. In this study, synergistic administration of L-egt and metformin to diabetic rats improves body weight. This suggests that this treatment regimen could halt some metabolic derangements associated with muscle wasting and loss of adipose tissues, to reduce weight loss during diabetes.

In this study, liver hypertrophy was seen in the DC rats compared to the NC rats, and this was in accordance with other studies where increased liver weight was reported in diabetic rats (Ayepola et al., 2013, Zhang et al., 2019). The increased liver weight may result from IR and hypertriglyceridemia that promote influx and accumulation of fatty acids into the liver cells (Mohamed et al., 2016, Zhang and Lu, 2015). Administration of L-egt, with or without metformin, to diabetic rats significantly reduced liver hypertrophy, suggesting that L-egt may reduce the influx of fatty acids into the liver or promote $\beta$ oxidation of fatty acids in the hepatic mitochondrial. Thus, preventing excessive accumulation of fatty acids and triacylglycerol in the liver. Effective regulation of fasting and postprandial blood glucose level plays a significant role in reducing diabetes-induced organ damage. In this study, co-administration of Legt with metformin to diabetic rats enhanced the antihyperglycemic efficacy of metformin (a conventional drug used in the management of type-2 diabetes). This could be responsible for the reduced blood glucose observed in the diabetic rat. Also, the reduced IR assessed by HOMA-IR in the DEM group indicates improved insulin sensitivity and glycemic control. Metformin reduces blood glucose by increasing insulin sensitivity and reducing hepatic gluconeogenesis (Foretz et al., 2019). The hepatoprotective effect of L-egt may improve the antihyperglycemic efficacy of metformin because the liver is a major site of metformin activity where it reduces hepatic lipogenesis and improves fatty acid oxidation (Zheng et al., 2015). Thus, L-egt supplementation during metformin therapy may enhance glycemic control and prevent hyperglycemia-induced liver damage.

Liver enzymes, including Alanine aminotransferase (ALT), Aspartate aminotransferase (AST), and alkaline phosphatase (ALP), are common biomarkers used to evaluate liver injury. These enzymes help catalyze crucial chemical reactions (e.g., amino transfer) and their serum concentration increases during liver damage (Kwo et al., 2017, Fu et al., 2020). An increase in serum aminotransferase is mostly reported in type-2 diabetes due to membrane damage in the hepatocytes, while increased ALT level may also indicate fatty liver resulting from reduced insulin sensitivity by the hepatocytes (Mandal et al., 2018, Islam et al., 2020). The increased serum level of ALP, AST, and ALT recorded in this study indicate significant liver injury in the DC rats. Interestingly, L-egt with or without metformin attenuated liver damage, as evidenced by reduced serum level of liver enzymes. This result suggests hepatoprotective function, which 
may result from the ability of L-egt to enhance membrane integrity in the hepatocytes to reduce leakage of liver enzymes into circulation. Notably, there was no significant change in the serum level of liver enzymes in the NE rats when compared with NC rats suggesting that L-egt does not alter liver functions in the normal rats and provides further credence to its safety evaluation.

In this study, hypertriglyceridemia observed in DC rats indicates a substantial alteration in fatty acid metabolism, which is another risk factor for diabetic complications. This result is in accordance with similar studies that reported a significant association between increased serum triglycerides (TG) and type-2 diabetes (Thambiah et al., 2016, Rašković et al., 2019). The altered metabolism increased serum TG, which promotes the influx of free fatty acids into the systemic circulation with subsequent infiltration into the hepatocytes with resultant steatosis or NAFLD. Besides, IR can inhibit lipoprotein lipase (LPL), which enhances the mobilization of TG to skeletal muscles and adipose tissue, thereby reducing the TG clearance from circulation (Arguello et al., 2015, Jiang et al., 2013). From this study, the significant reduction in serum and liver TG in diabetic animals treated with L-egt alone, or in combination with metformin may result from the inhibition of de novo biosynthesis as well as enhance lipoprotein lipase activity to increase the TG clearance from the circulation or thereby reducing the risk of fatty liver and related injuries (Alves-Bezerra and Cohen, 2017, Kawano and Cohen, 2013). Furthermore, the reduced TG infiltration into the hepatocyte in the L-egt treated groups may result from the downregulation of SREBP1c and FAS genes that promote TG synthesis.

Chronic hyperglycemia has been reported to increase free radical production by the neutrophils and activated kupffer cells. Elevated free radicals (e.g., ROS, $\mathrm{H}_{2} \mathrm{O}_{2}, \mathrm{OH}^{-}$) compromises the integrity of the cells via lipid peroxidation in the cell membrane, upregulate inflammatory signals and induce hepatic apoptosis as well as irreversible damage to other biomolecules in the body (e.g., protein, RNA). These biochemical events may result in significant structural and functional damage in the liver (Masarone et al., 2018, Lucchesi et al., 2015). In the present study, the reduced MDA in the L-egt treated groups suggests that L-egt may inhibit oxidative degradation of the lipid bilayers in the cell membrane to enhance cellular integrity and the viability of membrane proteins that helps in cellular communication. The increased antioxidant enzymes (SOD, CAT, and GSH) work synergistically to reduce the deleterious effect of free radicals in the tissues. An increased SOD level in the liver homogenates facilitates the detoxification of free radicals by enhancing the conversion of superoxide into hydrogen peroxide, while CAT helps to neutralize the free radicals by degrading hydrogen peroxide into water and oxygen molecule. Increased GSH reduces hydrogen peroxides to water and other lipid peroxides to alcohol, usually in the mitochondrial and cytosol (Ighodaro and Akinloye, 2018). In this study, administration of L-egt, with or without metformin, increased the production and efficacy of these antioxidant enzymes in diabetic rats, thereby protects against oxidative damage, enhance cell integrity, and complement the efficacy of antioxidant enzymes against ROS. The improved antioxidant defense system observed in the L-egt treated group may result from the upregulation of Sirt1 and Nrf2 genes, which are the major transcription factors in the antioxidant signaling pathways. Nrf2 is a master regulator of cellular antioxidant response that stimulates the production of phase II cytoprotective antioxidant genes (e.g., heme-oxygenase-1 and 
NAD (P)H oxidase-1) and ROS-detoxifying enzyme (e.g., GSH, SOD, CAT GPx) to mediate redox balance (Ma, 2013, Tonelli et al., 2017). Also, Sirt1 exerts a wide variety of biological functions, acting as a metabolic sensor that regulates lipid metabolism, inhibits free radical production from the respiratory chain, increases the detoxification of ROS by activating antioxidant enzymes, and downregulates the NFkB signaling pathway (Li, 2014, Singh et al., 2018).

The suppression of inflammatory molecules in the liver cells in response to tissue injury has attracted significant interest in managing liver disease. Low-grade chronic inflammation in metabolic diseases, IR, and their deleterious effect on liver function have been reported in previous studies (Ning et al., 2015, Hou et al., 2018). The concentration of TNF-a (a proinflammatory cytokine) varies directly with the severity of the liver disease (Seo et al., 2013, Yang and Seki, 2015), while MCP-1 and TGF- $\beta 1$ are responsible for monocyte activation, fatty acid accumulation, and fibrosis in the liver cells (Mandrekar et al., 2011, Nair and Nath, 2020). Taken together, the reduced inflammatory and fibrotic markers in the liver homogenates of diabetic animals treated with L-egt, with or without metformin showed that this treatment regimen may prevent hepatic inflammation and fibrosis, immune cell activation and apoptosis, as well as macrophage infiltration into the hepatocytes to reduce structural and functional damage (Gehrke and Schattenberg, 2020, Del Campo et al., 2018). This result is further supported by the downregulation of NF-kB (a major transcription factor in the inflammatory pathway) and TGF- $\beta 1$ gene expression in the L-egt treated groups. It has been established that activation of NF-kB causes the transcription of several genes that mediate cellular inflammation, while L-ergothioneine has been demonstrated to modulate NF-kB signaling to attenuate hepatic injury (Zeng et al., 2016, Salama and Omar, 2021). Thus, L-egt may protect against liver injury associated with type-2 diabetes by reducing hepatic inflammation and fibrosis

The histopathological examination of liver tissue provides essential information on the structural integrity of the liver. The disruption of the hepatic sinusoids and congested central vein with mild degeneration of the hepatocytes observed in the DC rats suggests significant liver damage. This result is consistent with other studies (Rodríguez et al., 2018, Ogar et al., 2019). However, improvement in the structural integrity of the diabetic liver treated with L-egt and metformin can be associated with the hepato-protective function of this treatment regimen. The regular appearance of hepatic sinusoids and central veins in the DEM rats may enhance adequate blood supply from the hepatic artery and portal vein towards the central veins, thus preventing ischemic cell death.

\section{Conclusion}

In conclusion, this study showed that L-egt can alleviate oxidative damage by upregulating Sirt1/Nrf2 expression and its downstream antioxidant molecules, downregulate NF-kB and TGF- $\beta 1$ expression to reduce hepatic inflammation and fibrosis as well as reduce SREBP1c and FAS expression to lower hypertriglyceridemia and attenuate hepatocyte fatty acid accumulation to protect liver function during diabetes. Thus, supplementation of L-egt could be used as an adjuvant regimen with metformin therapy in the early stage of diabetes to prevent the development or alter the progression of liver complications associated with diabetes. However, further studies to evaluate the status of L-egt transporters and protein 
expression of these transcription factors are required to provide the detailed mechanism of action of Legt.

\section{Declarations}

Acknowledgements: The authors thank Dr. Jean-Claude Yadan from Tetrahedron (Parc Technologique Biocitech 102 avenue Gaston Roussel, Romainville, F93230, France) for providing pure L-ergothioneine used in this study. The authors also acknowledge the assistance received from the Biomedical Resource Unit, Westville Campus, University of KwaZulu-Natal (UKZN).

Ethical approval: All animal and experimental procedures were approved by the Animal Research Ethics Committee (AREC) of the University of KwaZulu-Natal, Durban, South Africa (Ethic number: AREC/006/019D).

Consent to participate: Not applicable

Consent to publish: Not applicable

Author contribution statement: AD: conceive and designed the research, conducted experiment, wrote manuscript, acquired fund MC: Revision of the manuscript, supervision and acquire fund; AN: designed the experiment, revise initial draft of the manuscript and supervision. All authors read and approved the manuscript, and all data were generated in-house and that no paper mill was used.

Funding: This work was supported by the College of Health Science (CHS), University of KwaZulu-Natal, South Africa (grant number: 640997).

Competing interest: The authors have no conflicts of interest to declare that are relevant to the content of this article.

\section{References}

1. AEBI H (1984) Catalase in vitro. Methods Enzymol 105:121-126

2. ALVES-BEZERRA M, COHEN DE (2017) Triglyceride Metabolism in the Liver. Comprehensive Physiology 8:1-8

3. ARGUELLO G, BALBOA E, ARRESE M, ZANLUNGO S (2015) Recent insights on the role of cholesterol in non-alcoholic fatty liver disease. Biochim Biophys Acta 1852:1765-1778

4. ARRESE M, BARRERA F, TRIANTAFILO N, ARAB JP (2019) Concurrent nonalcoholic fatty liver disease and type 2 diabetes: diagnostic and therapeutic considerations. Expert Rev Gastroenterol Hepatol 13:849-866

5. AYEPOLA OR, CHEGOU, N. N., BROOKS, N. L. \& OGUNTIBEJU OO (2013) Kolaviron, a Garcinia biflavonoid complex ameliorates hyperglycemia-mediated hepatic injury in rats via suppression of inflammatory responses. BMC Complement Altern Med 13:363 
6. AZEEM U, SHRI, R. \& DHINGRA GS (2021) In Vitro and In Vivo Antihyperglycemic Activities of Medicinal Mushrooms (Agaricomycetes) from India. Int J Med Mushrooms 23:29-41

7. CARBONERO A, PALOMA G, GONZALO F, OLGA C, RENATO I, ANABEL, U., JOSÉ, A. \& JUAN B (2019) Ergothioneine Rich Agaricus Bisporus Extracts Decreases Lipid Accumulation Induced by Oleic Acid in Hepg2 Cells: Possible Implications in the Treatment of Nonalcoholic Liver Fatty Disease. J food Sci Nutr 5:049

8. CHOUDHURY H, JING PANDEY,MHUA,CK,MUN,CS, KONG JK, YEE LERN,LY,ASHRAF,NA,KIT,SW, GORAIN TS,PICHIKA,MR, B. \& KESHARWANI P (2017) An update on natural compounds in the remedy of diabetes mellitus: A systematic review. Journal of traditional complementary medicine 8:361-376

9. D'ONOFRIO N, CASALE SERVILLO,LGIOVANE,A, VITIELLO R, PAOLISSO MMARFELLA,R, G. \& BALESTRIERI ML (2016) Ergothioneine oxidation in the protection against high-glucose induced endothelial senescence: Involvement of SIRT1 and SIRT6. Free Radic Biol Med 96:211-222

10. DE GREGORIO E, COLELL A, MORALES A, MARÍ M (2020) Relevance of SIRT1-NF-KB Axis as Therapeutic Target to Ameliorate Inflammation in Liver Disease. Int J Mol Sci 21:3858-3882

11. DEL CAMPO JA, GALLEGO P, GRANDE L (2018) Role of inflammatory response in liver diseases: Therapeutic strategies. World journal of hepatology 10:1-7

12. ELLMAN GL (1959) Tissue sulfhydryl groups. Arch Biochem Biophys 82:70-77

13. EY J, SCHÖMIG E, TAUBERT D (2007) Dietary Sources and Antioxidant Effects of Ergothioneine. J Agric Food Chem 55:6466-6474

14. FARGHALI H, KEMELO, M. K. \& CANOVÁ NK (2019) SIRT1 Modulators in Experimentally Induced Liver Injury. Oxidative Medicine and Cellular Longevity, 2019, 8765954

15. FORETZ M, GUIGAS B, VIOLLET B (2019) Understanding the glucoregulatory mechanisms of metformin in type 2 diabetes mellitus. Nature Reviews Endocrinology 15:569-589

16. FU S, WU D, JIANG W, LI J, JIA LONG,J, C. \& ZHOU T (2020) Molecular Biomarkers in Drug-Induced Liver Injury: Challenges and Future Perspectives. Front Pharmacol 10:1667-1667

17. GEHRKE N, SCHATTENBERG JM (2020) Metabolic Inflammation-A Role for Hepatic Inflammatory Pathways as Drivers of Comorbidities in Nonalcoholic Fatty Liver Disease? Gastroenterology 158:1929-1947.e6

18. GOTHAI S, GANESAN P, PARK S-Y, FAKURAZI S, CHOI D-K, ARULSELVAN P (2016) Natural PhytoBioactive Compounds for the Treatment of Type 2 Diabetes: Inflammation as a Target. Nutrients 8:461

19. GUIJARRO MV, ARUOMA INDART,A, VIANA OI, M. \& BONET B (2002) Effects of ergothioneine on diabetic embryopathy in pregnant rats. Food chemical toxicology: an international journal published for the British Industrial Biological Research Association 40:1751-1755

20. HALLIWELL B, CHEAH, I. K. \& TANG RMY (2018) Ergothioneine - a diet-derived antioxidant with therapeutic potential. FEBS Lett 592:3357-3366 
21. HAZLEHURST JM, WOODS C, MARJOT T, COBBOLD JF, TOMLINSON JW (2016) Non-alcoholic fatty liver disease and diabetes. Metabolism 65:1096-1108

22. HOU B, ZHAO Y, QIANG G, YANG X, XU C, CHEN X, WANG LIU,C, ZHANG X, L. \& DU G (2018) Puerarin Mitigates Diabetic Hepatic Steatosis and Fibrosis by Inhibiting TGF- $\beta$ Signaling Pathway Activation in Type 2 Diabetic Rats. Oxid Med Cell Longev, 2018, 4545321

23. IGHODARO OM, AKINLOYE OA (2018) First line defence antioxidants-superoxide dismutase (SOD), catalase (CAT) and glutathione peroxidase (GPX): Their fundamental role in the entire antioxidant defence grid. Alexandria Journal of Medicine 54:287-293

24. ISLAM S, RAHMAN S, HAQUE T, AHMED SUMON,AH, A. M. \& ALI N (2020) Prevalence of elevated liver enzymes and its association with type 2 diabetes: A cross-sectional study in Bangladeshi adults. 3 , e00116

25. JADEJA RN, DEVKAR UPADHYAY,KK, R. V. \& KHURANA S (2016) Naturally Occurring Nrf2 Activators: Potential in Treatment of Liver Injury. Oxidative Medicine and Cellular Longevity, 2016, 3453926

26. JEONG YU, PARK YJ (2020) Ergosterol Peroxide from the Medicinal Mushroom Ganoderma lucidum Inhibits Differentiation and Lipid Accumulation of 3T3-L1 Adipocytes. Int J Mol Sci, 21

27. JIANG ZG, ROBSON SC, YAO Z (2013) Lipoprotein metabolism in nonalcoholic fatty liver disease. Journal of biomedical research 27:1-13

28. KAWANO Y, COHEN DE (2013) Mechanisms of hepatic triglyceride accumulation in non-alcoholic fatty liver disease. Journal of gastroenterology 48:434-441

29. KO HJ, KIM J, AHN M, LEE KIM,JH, G. S. \& SHIN T (2021) Ergothioneine alleviates senescence of fibroblasts induced by UVB damage of keratinocytes via activation of the Nrf2/HO-1 pathway and HSP70 in keratinocytes. Exp Cell Res 400:112516

30. KWO PY, COHEN SM, LIM JK (2017) ACG Clinical Guideline: Evaluation of Abnormal Liver Chemistries. Am J Gastroenterol 112:18-35

31. LI H (2014) Sirtuin 1 (SIRT1) and Oxidative Stress. In: LAHER I (ed) Systems Biology of Free Radicals and Antioxidants. Springer Berlin Heidelberg, Berlin

32. LINDEQUIST U, HAERTEL B (2020) Medicinal Mushrooms for Treatment of Type 2 Diabetes: An Update on Clinical Trials. Int J Med Mushrooms 22:845-854

33. LIVAK KJ, SCHMITTGEN TD (2001) Analysis of relative gene expression data using real-time quantitative PCR and the 2(-Delta Delta C(T)) Method. Methods 25:402-408

34. LO HC, HSU TH, LEE CH (2020) Extracellular Polysaccharopeptides from Fermented Turkey Tail Medicinal Mushroom, Trametes versicolor (Agaricomycetes), Mitigate Oxidative Stress, Hyperglycemia, and Hyperlipidemia in Rats with Type 2 Diabetes Mellitus. Int J Med Mushrooms 22:417-429

35. LOZANO I, VAN DER WERF R, BIETIGER W, SEYFRITZ E, PERONET C, PINGET M, JEANDIDIER N, MARCHIONI MAILLARD,E, E., SIGRIST, S. \& DAL S (2016) High-fructose and high-fat diet-induced disorders in rats: impact on diabetes risk, hepatic and vascular complications. Nutr Metab (Lond), 13, 15

Page $16 / 23$ 
36. LUCCHESI AN, CASSETTARI, L. L. \& SPADELLA CT (2015) Alloxan-Induced Diabetes Causes Morphological and Ultrastructural Changes in Rat Liver that Resemble the Natural History of Chronic Fatty Liver Disease in Humans. Journal of Diabetes Research, 2015, 494578

37. MA F, JIANG WU,J, HUANG Z, JIA W, SUN Y, W. \& WU H (2019) P53/NRF2 mediates SIRT1's protective effect on diabetic nephropathy. Biochimica et Biophysica Acta (BBA) - Molecular Cell Research 1866:1272-1281

38. MA Q (2013) Role of nrf2 in oxidative stress and toxicity. Annu Rev Pharmacol Toxicol 53:401-426

39. MAGALHÃES DA, KUME WT, CORREIA FS, QUEIROZ TS, ALLEBRANDT NETO EW, SANTOS MPD, KAWASHITA, N. H. \& FRANÇA SA (2019) High-fat diet and streptozotocin in the induction of type 2 diabetes mellitus: a new proposal. An Acad Bras Cienc 91:e20180314

40. MANDAL A, BHATTARAI B, KAFLE P, KHALID M, LAMICCHANE JONNADULA,SK, KANTH J, R. \& GAYAM V (2018) Elevated Liver Enzymes in Patients with Type 2 Diabetes Mellitus and Non-alcoholic Fatty Liver Disease. Cureus, 10, e3626-e3626

41. MANDREKAR P, AMBADE A, LIM A, SZABO G, CATALANO D (2011) An essential role for monocyte chemoattractant protein-1 in alcoholic liver injury: regulation of proinflammatory cytokines and hepatic steatosis in mice. Hepatology 54:2185-2197

42. MARKLUND SL (1985) Product of extracellular-superoxide dismutase catalysis. FEBS Lett 184:237239

43. MASARONE M, ROSATO V, DALLIO M, AGLITTI GRAVINA,AG, LOGUERCIO A, C., FEDERICO, A. \& PERSICO M (2018) Role of Oxidative Stress in Pathophysiology of Nonalcoholic Fatty Liver Disease. Oxidative Medicine and Cellular Longevity, 2018, 9547613

44. MATTHEWS DR, RUDENSKI HOSKER,JP, TREACHER AS,NAYLOR,BA, D. F. \& TURNER RC (1985) Homeostasis model assessment: insulin resistance and beta-cell function from fasting plasma glucose and insulin concentrations in man. Diabetologia 28:412-419

45. MCKINLEY S, WILLOUGHBY D (2014) Effectiveness of antioxidant nutraceuticals in attenuating canonical NF- $\mathrm{kb}$ signaling in human skeletal muscle resulting from exercise-induced inflammation and oxidative stress. J Nutr Health Food Eng 1:214-221

46. MKHWANAZI BN, VAN SERUMULA,MR,MYBURG,RB, HEERDEN FR, MUSABAYANE CT (2014) Antioxidant effects of maslinic acid in livers, hearts and kidneys of streptozotocin-induced diabetic rats: effects on kidney function. Ren Fail 36:419-431

47. MOHAMED J, NAZRATUN NAFIZAH AH, ZARIYANTEY, A. H. \& BUDIN SB (2016) Mechanisms of Diabetes-Induced Liver Damage: The role of oxidative stress and inflammation. Sultan Qaboos Univ Med J 16:e132-e141

48. NAIR B, NATH LR (2020) Inevitable role of TGF- $\beta 1$ in progression of nonalcoholic fatty liver disease. J Recept Signal Transduct Res 40:195-200

49. NING C, LIU L, LV G, YANG Y, ZHANG Y, WANG YU,R, Y. \& ZHU J (2015) Lipid metabolism and inflammation modulated by Vitamin $D$ in liver of diabetic rats. Lipids Health Dis 14:31 
50. OGAR I, NNA EGBUNG,GE, V. U., ATANGWHO, I. J. \& ITAM EH (2019) Hyptis verticillata attenuates dyslipidaemia, oxidative stress and hepato-renal damage in streptozotocin-induced diabetic rats. Life Sci 219:283-293

51. PANDEY A, CHAWLA S, GUCHHAIT P (2015) Type-2 diabetes: Current understanding and future perspectives. 67, 506-513

52. PERRY RJ, KURSAWE CAMPOREZ,JG, TITCHENELL R, ZHANG PM, PERRY D, JURCZAK CJ, HAN MJ,ABUDUKADIER,A, ZHANG MS, YANG XM,RUAN,HB, KAECH XCAPRIO,S, BIRNBAUM SM,SUL,HS, M. J., DAVIS, R. J., CLINE, G. W., PETERSEN, K. F. \& SHULMAN GI (2015) Hepatic acetyl CoA links adipose tissue inflammation to hepatic insulin resistance and type 2 diabetes. Cell 160:745-758

53. RAŠKOVIĆ A, ĆUĆUZ V, TOROVIĆ L, TOMAS A, GOJKOVIĆ-BUKARICA L, MILIJAŠEVIĆ ĆEBOVIĆ,T, STILINOVIĆ B, N. \& CVEJIĆ HOGERVORST, J. 2019. Resveratrol supplementation improves metabolic control in rats with induced hyperlipidemia and type 2 diabetes. Saudi pharmaceutical journal: SPJ : the official publication of the Saudi Pharmaceutical Society, 27, 1036-1043

54. RENA G, HARDIE, D. G. \& PEARSON ER (2017) The mechanisms of action of metformin. Diabetologia 60:1577-1585

55. RODRÍGUEZ V, PLAVNIK L, TOLOSA DE TALAMONI N (2018) Naringin attenuates liver damage in streptozotocin-induced diabetic rats. Biomed Pharmacother 105:95-102

56. ROMANO A, FRIULI M, COCO DEL, LONGO L, VERGARA S, BOCCIO DDEL, VALENTINUZZI P, CICALINI S, FANIZZI I, F. P., GAETANI, S. \& GIUDETTI, A. M (2021) Chronic Oleoylethanolamide Treatment Decreases Hepatic Triacylglycerol Level in Rat Liver by a PPARY/SREBP-Mediated Suppression of Fatty Acid and Triacylglycerol Synthesis. Nutrients, 13

57. SALAMA SA, OMAR HA (2021) Modulating NF-KB, MAPK, and PI3K/AKT signaling by ergothioneine attenuates iron overload-induced hepatocellular injury in rats. J Biochem Mol Toxicol, e22729

58. SEO YY, CHO YK, BAE JC, SEO MH, PARK SE, RHEE EJ, PARK, C. Y., OH, K. W., PARK, S. W. \& LEE WY (2013) Tumor Necrosis Factor-a as a Predictor for the Development of Nonalcoholic Fatty Liver Disease: A 4-Year Follow-Up Study. Endocrinol Metab (Seoul) 28:41-45

59. SINGH CK, CHHABRA G, NDIAYE MA, GARCIA-PETERSON LM, MACK, N. J. \& AHMAD N (2018) The Role of Sirtuins in Antioxidant and Redox Signaling. Antioxid Redox Signal 28:643-661

60. SONG TY, YANG NC, CHEN CL, THI TLV (2017) Protective Effects and Possible Mechanisms of Ergothioneine and Hispidin against Methylglyoxal-Induced Injuries in Rat Pheochromocytoma Cells. Oxid Med Cell Longev, 2017, 4824371

61. SRINIVASAN K, VISWANAD B, ASRAT L, KAUL CL, RAMARAO P (2005) Combination of high-fat dietfed and low-dose streptozotocin-treated rat: a model for type 2 diabetes and pharmacological screening. Pharmacol Res 52:313-320

62. TANG RMY, CHEAH, I. K.-M., YEW TSK, HALLIWELL B (2018) Distribution and accumulation of dietary ergothioneine and its metabolites in mouse tissues. Sci Rep 8:1601

63. TANG Y, MASUO Y, SAKAI Y, WAKAYAMA T, SUGIURA T, HARADA R, KOMURA FUTATSUGI,A, NAKAMICHI T, USUMI NSEKIGUCHI,HSUTOH,K, ISEKI K, S., KANEKO, S. \& KATO Y (2016) Localization 
of Xenobiotic Transporter OCTN1/SLC22A4 in Hepatic Stellate Cells and Its Protective Role in Liver Fibrosis. J Pharm Sci 105:1779-1789

64. THAMBIAH SC, SAMSUDIN IN, ZAHARI GEORGE,E, SHAM SY, LEE HM, MUHAMAD MA, HUSSEI Z, MOHD NOOR, N. \& MOHAMAD M (2016) Relationship between dyslipidaemia and glycaemic status in patients with Type 2 diabetes mellitus. Malays J Pathol, 38, $123-30$

65. TONELLI C, CHIO, I. I. C, TUVESON DA (2017) Transcriptional Regulation by Nrf2. Antioxid Redox Signal 29:1727-1745

66. WANG Z, YUAN S, LI Y, ZHANG Z, TANG XIAO,W, LIU DYE,K, WANG Z, ZHENG C, NIE Y, H. \& CHEN H (2018) Regulation on SIRT1-PGC-1 a/Nrf2 pathway together with selective inhibition of aldose reductase makes compound hr5F a potential agent for the treatment of diabetic complications. Biochem Pharmacol 150:54-63

67. WILLIAMSON RD, MANNA MCCARTHY,FP, GROARKE S, KELL E, D. B., KENNY, L. C. \& MCCARTHY CM (2020) L-(+)-Ergothioneine Significantly Improves the Clinical Characteristics of Preeclampsia in the Reduced Uterine Perfusion Pressure Rat Model. 75, 561-568

68. WILSON RD, ISLAM MS (2012) Fructose-fed streptozotocin-injected rat: an alternative model for type 2 diabetes. Pharmacol Rep 64:129-139

69. XU LN, JIN YIN,LH, QI Y, HAN Y, XU X, Y. W., LIU, K. X., ZHAO, Y. Y. \& PENG JY (2020) Effect and possible mechanisms of dioscin on ameliorating metabolic glycolipid metabolic disorder in type-2diabetes. Phytomedicine 67:153139

70. YANG YM, SEKI E (2015) TNFa in liver fibrosis. Current pathobiology reports 3:253-261

71. ZENG T, HE ZHOU,J, WU LZHENG,JCHEN,L, C. \& XIA W (2016) Blocking Nuclear Factor-Kappa B Protects against Diet-Induced Hepatic Steatosis and Insulin Resistance in Mice. PLOS ONE 11:e0149677

72. ZHANG Q-Q, LU L-G (2015) Nonalcoholic Fatty Liver Disease: Dyslipidemia, Risk for Cardiovascular Complications, and Treatment Strategy. Journal of clinical translational hepatology 3:78-84

73. ZHANG WQ, ZHAO TT, GUI DK, GAO CL, HUANG GU,JL,GAN,WJ, XU W, Y., ZHOU, H., CHEN, W. N., LIU, Z. L. \& XU YH (2019) Sodium Butyrate Improves Liver Glycogen Metabolism in Type 2 Diabetes Mellitus. J Agric Food Chem 67:7694-7705

74. ZHENG J, WOO S-L, HUO HU,XBOTCHLETT,RCHEN,L, Y. \& WU C (2015) Metformin and metabolic diseases: a focus on hepatic aspects. Frontiers of medicine 9:173-186

75. ZOPPINI G, TARGHER FEDELI,UGENNARO,NSAUGO,M, G. \& BONORA E (2014) Mortality from chronic liver diseases in diabetes. Am J Gastroenterol 109:1020-1025

\section{Figures}



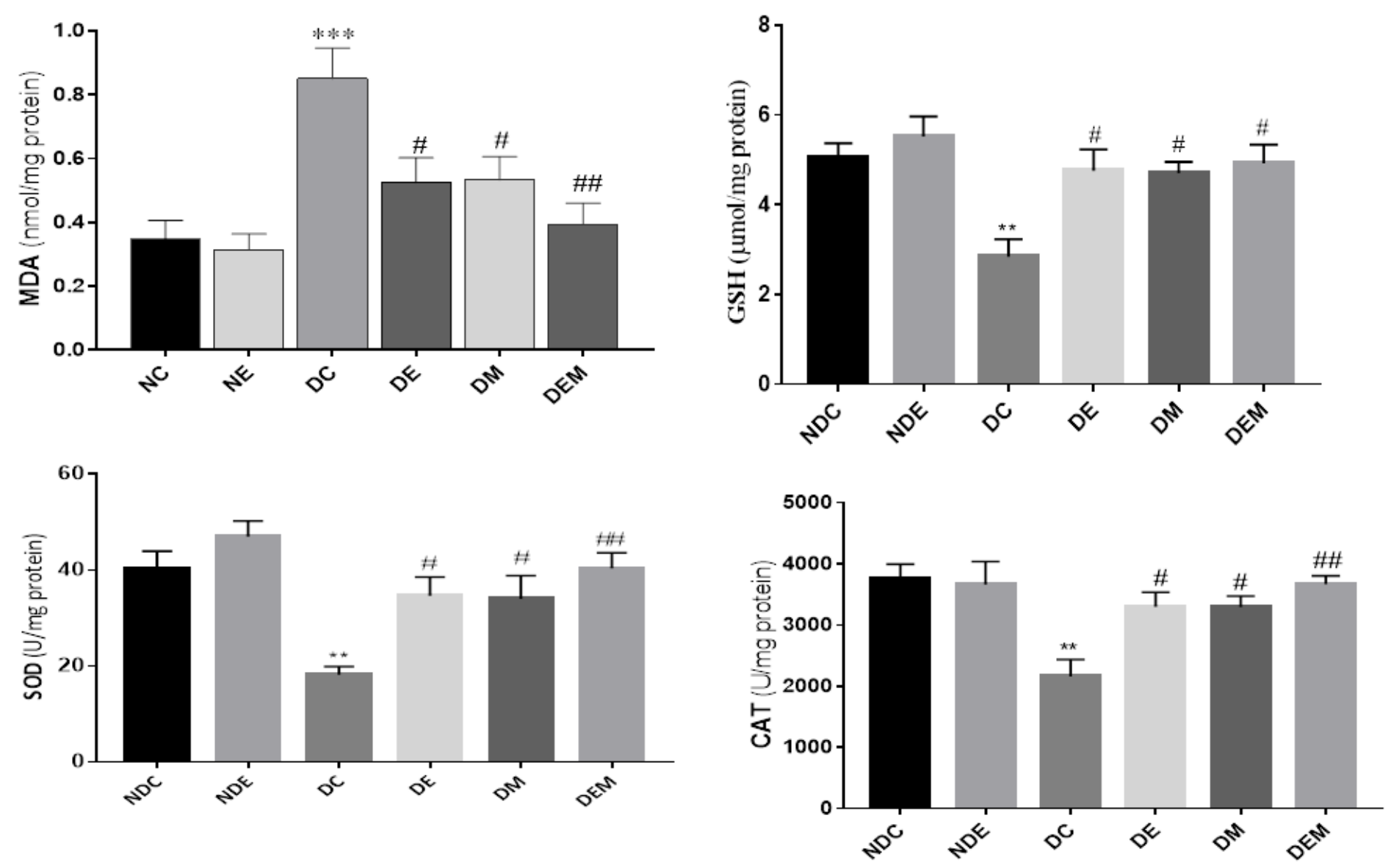

\section{Figure 1}

a: MDA levels in L-egt, with or without metformin, treated T2DM rats b: SOD levels in L-egt, with or without metformin, treated T2DM rats c: GSH levels in L-egt, with or without metformin, treated T2DM rats d: CAT levels in L-egt, with or without metformin, treated T2DM rats 

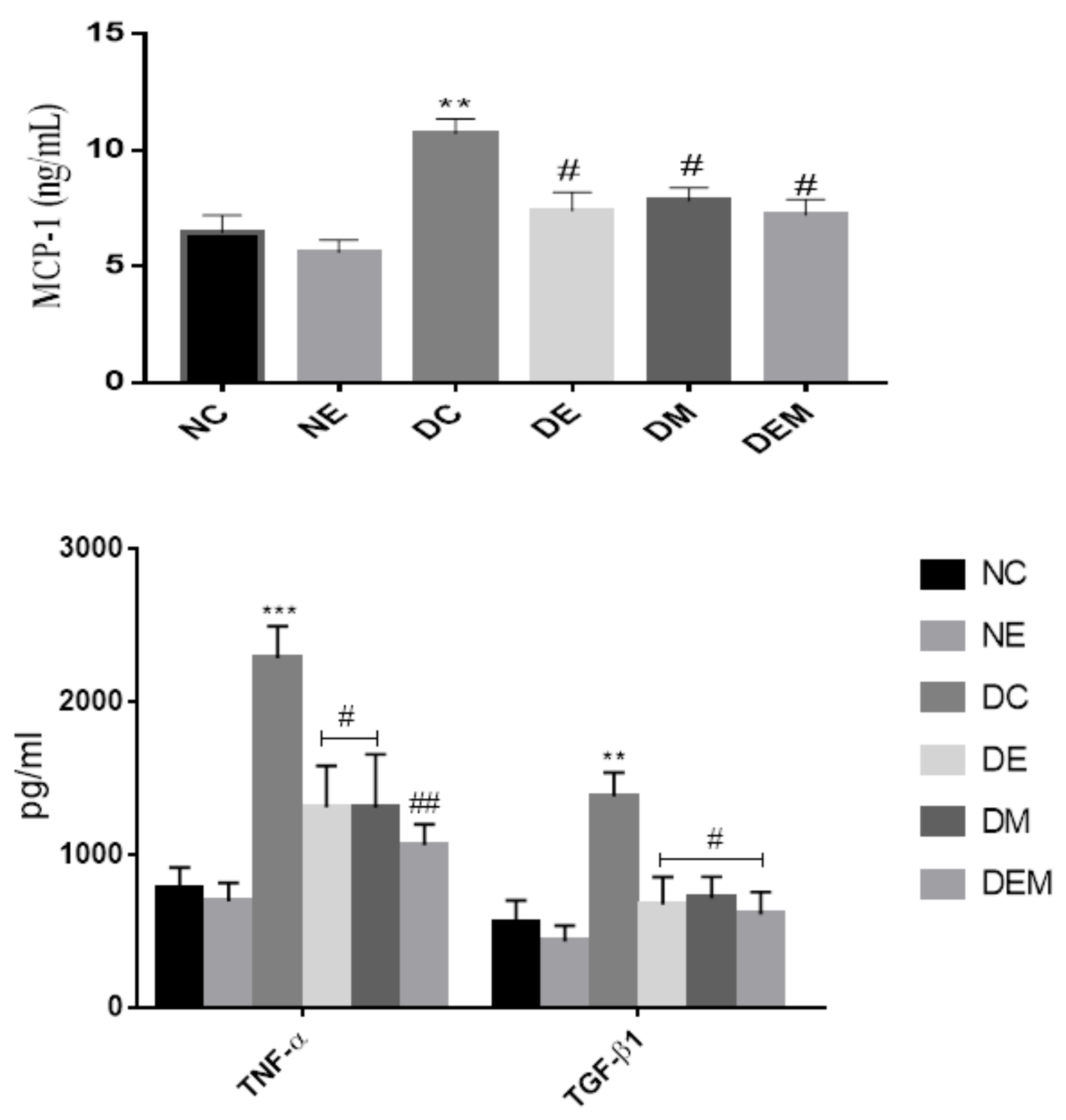

Figure 2

a-b: Effect of L-egt, with or without metformin, on liver inflammatory biomarkers (A) MCP1, (B) TNF-a and TGF- $\beta 1$ in T2DM-rats. ${ }^{* \star *} p<0.001,{ }^{*} p<<0.01$ vs NC; $\# \# p<0.01, \# p<0.05$ vs DC. $(n=5)$. NC= Nondiabetic control; $\mathrm{NE}=$ nondiabetic plus L-egt; $\mathrm{DC}=$ diabetic control; $\mathrm{DE}=$ diabetic plus $\mathrm{L}$-egt; $\mathrm{DM}=$ diabetic plus metformin and $\mathrm{DEM}=$ diabetic plus L-egt plus metformin. (supplemental data). 

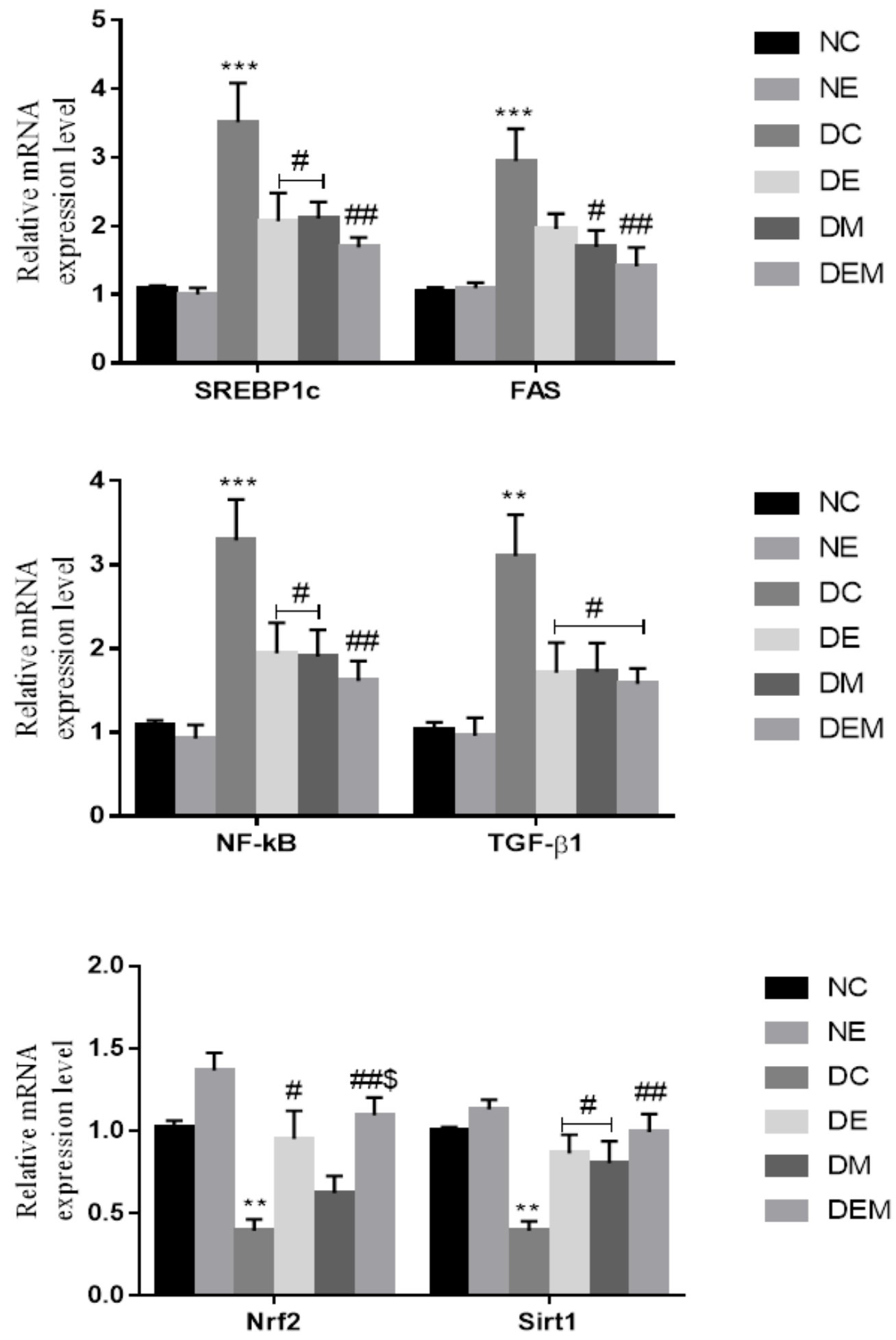

Figure 3

a-c: Relative mRNA expression level of (A) SREBP1c and FAS (B), NF-kB and TGF- $\beta 1$ (C), Nrf2 and Sirt1 relative gene expression in liver tissues of diabetic animals treated with L-egt with or without metformin for 7 weeks. ${ }^{\star \star *} p<0.001,{ }^{* \star} p<0.01$ vs NC groups; $\# p<0.05$, \#\#p<0.01 vs DC group and $\$ p<0.05$ vs DM $(n=5) . N C=$ Nondiabetic control; NE=nondiabetic plus L-egt; $D C=$ diabetic control; $D E=$ diabetic plus L-egt; $\mathrm{DM}=$ diabetic plus metformin and DEM= diabetic plus L-egt plus metformin (supplemental data). 


\section{Supplementary Files}

This is a list of supplementary files associated with this preprint. Click to download.

- Supplementaldata.xlsx 